\title{
Neutrophil to Lymphocyte Ratio Predicts Glucocorticoid Resistance in Polymyalgia Rheumatica: A Prospective Study
}

Claire Owen ( $\square$ claireowen85@gmail.com )

Austin Health https://orcid.org/0000-0002-2694-5411

Christopher McMaster

Austin Health

David FL Liew

Austin Health

Jessica L Leung

Austin Health

Andrew M Scott

Austin Health

Russell RC Buchanan

Austin Health

Research article

Keywords: polymyalgia rheumatica, neutrophils, lymphocytes, glucocorticoids, prognosis

Posted Date: February 26th, 2020

DOl: https://doi.org/10.21203/rs.2.24627/v1

License: (1) This work is licensed under a Creative Commons Attribution 4.0 International License.

Read Full License 


\section{Abstract}

Background Neutrophil to lymphocyte ratio (NLR) and platelet to lymphocyte ratio (PLR) have been found to correlate with disease activity in several rheumatic diseases, however their clinical and prognostic utility in polymyalgia rheumatica (PMR) remains unclear. This study evaluated the relationship between NLR and PLR, and disease activity and glucocorticoid resistance in PMR.

Methods Data for disease activity (PMR-AS) and full blood examination was obtained from a prospective observational cohort comprising newly diagnosed (2012 EULAR/ACR classification criteria), steroid-naïve PMR patients treated with low-dose glucocorticoid therapy (BSR guideline). Glucocorticoid resistance was defined as non-response to initial prednisolone dose $(15 \mathrm{mg} /$ day) or initial response followed by flare (PMR-AS $\geq 9.35$ or $\Delta \geq 6.6$ ) upon weaning prednisolone to $5 \mathrm{mg} /$ day. Univariable Bayesian linear regression analysis of the relationship between PMR-AS (baseline and mean) and NLR and PLR was performed. Predictors of glucocorticoid resistance were identified using a multivariable outcome model, with variables chosen based upon Bayesian model selection.

Results Of the 32 included patients, 16 (50\%) fulfilled the primary outcome measure of glucocorticoid resistance. These participants were older, typically female, and had higher baseline CRP than their glucocorticoid-responsive counterparts. A statistically significant relationship was identified between PMR-AS and both NLR (OR 28.1 [95\% Cl 1.6 - 54.7]) and PLR (OR 40.6 [95\% Cl 10.1 - 71.4]) at baseline, with PLR also found to correlate with disease activity during follow-up (OR 15.6 [95\% $\mathrm{Cl} 2.7-28.2]$ ). Baseline NLR proved to be a statistically significant predictor of glucocorticoid-resistant PMR (OR 14.01 [95\% Cl $1.49-278.06])$.

Conclusion Baseline NLR can predict glucocorticoid resistance in newly diagnosed PMR patients. Both NLR and PLR may be reliable biomarkers of disease activity in PMR.

\section{Background}

Polymyalgia rheumatica (PMR) is an inflammatory condition characterised by subacute-onset shoulder and hip pain, and stiffness. Oral glucocorticoids represent the treatment mainstay and whilst cessation of therapy is the ultimate goal, up to $50 \%$ of patients still require prednisolone $2-3$ years after diagnosis.(1) A higher baseline erythrocyte sedimentation rate (ESR) has been associated with disease relapse and lower probability of glucocorticoid discontinuation, however these findings have not been consistently reproduced.(2) To date, a reliable and readily accessible biomarker to measure disease activity and predict resistance to standardised low-dose glucocorticoid therapy has not been identified in PMR.

Neutrophil, platelet and lymphocyte counts undergo temporary changes in an active inflammatory state. Neutrophil to lymphocyte ratio (NLR) and platelet to lymphocyte ratio (PLR) can be calculated from a routine full blood examination (FBE) using the neutrophil count or platelet count divided by the lymphocyte count. In oncology, high NLR and PLR are associated with poor prognostic outcomes across a range of solid organ malignancies. $(3,4)$ These indices have similarly been found to correlate with 
disease activity in several rheumatic diseases including most recently PMR, but their role in predicting treatment response in this condition is less well-defined.(5-8)

In this study, we sought to prospectively characterize the relationship between NLR and PLR, and disease activity in patients with newly diagnosed PMR. The ability of NLR and PLR to predict resistance to standardised low-dose glucocorticoid therapy was also evaluated.

\section{Methods}

\section{Patients}

Patients with newly diagnosed, steroid-naïve PMR according to the 2012 EULAR/ACR classification criteria(9) were prospectively recruited from primary care, community and hospital rheumatology practices. Participants with symptoms suggestive of giant cell arteritis (GCA) including headache, jaw claudication, scalp tenderness or visual disturbance were excluded, along with cases of cancer within the past 5 years, neuromuscular disease, active infection, other inflammatory conditions (eg. rheumatoid arthritis [RA]) and chronic pain syndromes. The study was approved by the Austin Health Human Research Ethics Committee (HREC/14/Austin/158) prior to commencement and registered with the Australian New Zealand Clinical Trials Registry (trial identification ACTRN1261400696695).

Following written consent, demographic and clinical data were collected and the health assessment questionnaire-disability index (HAQ-DI) completed. A standard physical examination was undertaken including measurement of the shoulder range of motion (elevation of upper limb score). In order to measure disease activity using the PMR-activity score (PMR-AS)(10), participants marked a visual analogue scale indicating pain severity and the investigator provided a physician global assessment. Relevant differential diagnoses were excluded by testing creatine kinase, thyroid function, rheumatoid factor, anti-citrullinated peptide antibodies (ACPA) and anti-neutrophil cytoplasmic antibodies. FBE, Creactive protein (CRP) and erythrocyte sedimentation rate (ESR) were performed at baseline and each subsequent visit. A whole body ${ }^{18} \mathrm{~F}$-Fluorodeoxyglucose $\left({ }^{18} \mathrm{~F}-\mathrm{FDG}\right)$ positron emission tomography/computed tomography (PET/CT) scan (Gemini-TF64 or Ingenuity-TF128 PET/CT system; Phillips, Cleveland, $\mathrm{OH}, \mathrm{USA}$ ) from skull vertex to toes was also undertaken in all participants within 7 days of study enrolment.

\section{Treatment Protocol}

Participants were treated with a standardised schedule of low-dose glucocorticoid therapy as outlined in the British Society of Rheumatology (BSR) guideline.(11) Follow-up visits were scheduled at weeks 4, 8, $16,24,32$ and 46 (the minimum duration of this treatment protocol) and disease activity was recalculated. A PMR-AS score of $\geq 9.35$ in a patient who had achieved clinical remission (PMR-AS $<9.35$ ) or increase in PMR-AS by $\geq 6.6$ if the participant had been previously responding to treatment (PMR-AS $\geq 9.35$ but falling between successive visits) constituted the definition of disease relapse. When this occurred, the patient's prednisolone dose was increased to the previous higher dose for 4 weeks and an 
additional study visit performed to determine if the weaning schedule could be reinstated. If relapse occurred on more than two occasions, methotrexate was commenced as a steroid-sparing agent, and the participant was withdrawn from the study.

\section{Glucocorticoid Resistance}

Glucocorticoid resistance has been previously defined by Mori et al. in PMR as non-response to an initial prednisolone dose of $15 \mathrm{mg} /$ day or initial response followed by disease relapse upon weaning prednisolone to $5 \mathrm{mg} /$ day.(12) This definition was accordingly utilised as the primary outcome measure for glucocorticoid-resistant disease in our study.

\section{Statistical Analyses}

All statistical analyses were conducted using R version 3.5 .2 and the package rstanarm. $(13,14)$ Results for continuous variables are reported as mean \pm standard deviation or median (interquartile range). NLR and PLR, both right-skewed distributions, were log-transformed prior to analysis. Univariable Bayesian linear regression was used to analyse the relationship between NLR and PLR, and PMR-AS (baseline and mean). Several multivariable, Bayesian logistic regression models were compared using leave-one-out cross-validation - from the loo package(15) - to select predictors of resistance to low-dose glucocorticoid therapy. All outcomes are reported as odds ratios (OR) with $95 \%$ credible intervals (Cl).(16) Weakly-informative prior distributions were applied using student t-distribution with 7 degrees of freedom.

\section{Results}

Thirty-two of the 35 patients (91.4\%) recruited at baseline were suitable for inclusion in the final analysis (Table 1): one patient was excluded due to large vessel vasculitis on whole body PET/CT consistent with concomitant GCA; another patient's final diagnosis was revised to Parkinson's disease; and the third patient required a modified schedule of glucocorticoid therapy due to a history of prednisolone-induced central serous retinopathy.

Table 1 - Baseline demographic information: values are means \pm standard deviation, unless otherwise stated; median values are reported with $25 \%$ and $75 \%$ quartiles. 


\begin{tabular}{|ll|}
\hline Demographic & $\begin{array}{l}\text { Patients } \\
(\mathrm{n}=32)\end{array}$ \\
\hline Age (years) & $69.0 \pm 7.2$ \\
\hline Male & $17(53.1 \%)$ \\
\hline Caucasian & $31(96.9 \%)$ \\
\hline BMI (kg/m $\left.{ }^{2}\right)$ & $27.7 \pm 5.1$ \\
\hline Shoulder Pain & $32(100 \%)$ \\
\hline Hip Pain & $28(87.5 \%)$ \\
\hline Median EMS (mins) & $120(67.5-195)$ \\
\hline EULAR/ACR & $5.2 \pm 0.6$ \\
\hline Clinical Algorithm Score & \\
\hline Median CRP (mg/L) & $42.9(21.8-65.7)$ \\
\hline Median ESR (mm) & $47.5(30.5-68)$ \\
\hline PMR-AS & $74.2 \pm 35.7$ \\
\hline Median HAQ-DI & $1.7(1.1-2.1)$ \\
\hline
\end{tabular}

Abbreviations: BMI - body mass index; EMS - early morning stiffness; CRP - C-reactive protein; ESR erythrocyte sedimentation rate; PMR-AS - polymyalgia rheumatica-activity score; HAQ-DI - health assessment questionnaire-disability index.

Table 2 - Demographic comparison of glucocorticoid-resistant and -responsive patients: values are means \pm standard deviation, unless otherwise stated; median values are reported with $25 \%$ and $75 \%$ quartiles. 


\begin{tabular}{|lll|}
\hline Demographic & Glucocorticoid-Resistant $(\mathrm{n}=16)$ & $\begin{array}{l}\text { Glucocorticoid-Responsive } \\
(\mathrm{n}=16)\end{array}$ \\
\hline Age (years) & $71.9 \pm 7.0$ & $66.1 \pm 6.4$ \\
\hline $\begin{array}{l}\text { Gender: } \\
\text { - Female }\end{array}$ & $10(62.5 \%)$ & $5(31.25 \%)$ \\
\hline Male & $6(37.50 \%)$ & $11(68.75 \%)$ \\
\hline ESR $(\mathrm{mg} / \mathrm{L})$ & $59.3 \pm 46.0$ & $36.4 \pm 27.6$ \\
\hline PMR-AS & $50.7 \pm 28.6$ & $49.4 \pm 30.1$ \\
\hline Median WCC $\left(x 10^{9 / L}\right)$ & $85.3 \pm 35.3$ & $63.0 \pm 33.7$ \\
\hline Median neutrophils $\left(x 10^{9 / L}\right)$ & $5.5(4.9-6.73)$ & $7.95(7.0-9.23)$ \\
\hline Median lymphocyte $\left(x 10^{9 / L}\right)$ & $1.7(1.6-1.8)$ & $5.1(3.8-5.9)$ \\
\hline Median platelets $\left(x 10^{9 / L}\right)$ & $362(297-409)$ & $2.1(1.9-2.3)$ \\
\hline NLR (log-transformed) & $1.2 \pm 0.4$ & $332(264-448)$ \\
\hline PLR (log-transformed) & $5.37 \pm 0.37$ & $0.8 \pm 0.4$ \\
\hline
\end{tabular}

Abbreviations: CRP - C-reactive protein; ESR - erythrocyte sedimentation rate; PMR-AS - polymyalgia rheumatica-activity score; WCC - white cell count; NLR - neutrophil to lymphocyte ratio; PLR - platelet to lymphocyte ratio.

Glucocorticoid-resistant patients were older and typically female compared with their glucocorticoidresponsive counterparts. Baseline CRP, but not ESR, and disease activity (PMR-AS) were also higher. In terms of haematologic parameters, median white cell count (WCC), neutrophil and platelet counts were similar between the two groups, but the median lymphocyte count was lower in glucocorticoid-resistant patients (1.7 [1.6-1.8] cf. 2.1 [1.9-2.3]). Consequently, values for mean log-transformed NLR (1.20 \pm $0.48)$ and PLR $(5.37 \pm 0.37)$ were higher in this group than the glucocorticoid-responsive cohort (NLR 0.87 \pm 0.38 ; PLR $5.12 \pm 0.38$ ).

A statistically significant relationship was identified between disease activity (PMR-AS) and both NLR (OR 28.1 [95\% Cl 1.6-54.7]) and PLR (OR 40.6 [95\% Cl 10.1-71.4]) at baseline. During follow-up, PLR correlated with mean PMR-AS (OR 15.6 [95\% $\mathrm{Cl} 2.7-28.2]$ ), with a trend towards significance observed between NLR and mean PMR-AS (OR 10.1 [95\% Cl-0.9-21.3]).

Using Bayesian outcome modelling, baseline NLR was found to be a statistically significant predictor of glucocorticoid-resistant PMR during follow-up (OR 14.01 [95\% Cl 1.49-278.06]). A trend towards 
significance was also noted for baseline PLR (OR 5.02 [0.49-71.54]), along with age (OR 1.14 [95\% Cl 1.00-1.34]) and female sex (OR 2.98 [0.54-18.39]). There was no correlation between baseline CRP and glucocorticoid resistance after adjusting for demographic factors (OR 1.02 [95\% $\mathrm{Cl} 0.99-1.05]$ ). Figure 1 illustrates the relationship between baseline laboratory values for CRP, ESR and NLR, and glucocorticoidresistant PMR.

\section{Discussion}

In a cohort of 32 patients with newly diagnosed PMR, 16 (50\%) had a disease course characterized by resistance to low-dose glucocorticoid therapy, with baseline NLR proving a distinct predictor of this outcome. A trend towards significance was also observed between NLR and disease activity, with a statistically significant correlation found between PLR and PMR-AS both at baseline and during followup. All participants were steroid-naïve at study enrolment, glucocorticoid therapy thus having no bearing upon baseline haematologic parameters.

An increasing body of evidence now refutes historical opinion that PMR is a self-limiting, perpetually steroid-responsive entity. In a recent population-based study, around $25 \%$ of patients required more than 4 years of continuous prednisolone.(18) Significant morbidity is known to arise from this treatment paradigm whereby glucocorticoid monotherapy is advocated in the first instance and steroid-sparing agents are only initiated following recurrent relapse.(11, 19) On whole-body magnetic resonance imaging (MRI) however, a complete patient-reported response to prednisolone has been shown to correlate with an extra-capsular pattern of inflammation, thereby confirming the existence of discrete phenotypic differences between glucocorticoid-responsive and -resistant PMR cases.(20) Identification of baseline NLR as a predictor of later glucocorticoid-resistance in this study therefore has certain clinical relevance. Not only does it add to the paucity of recognized adverse prognostic indicators in this condition (such as female sex, peripheral joint involvement and high ESR)(2), but it also further characterizes the subset of patients with relapsing PMR whom may benefit from early disease modifying anti-rheumatic drug (DMARD) initiation.

Relying upon systemic markers of inflammation to assess disease activity in PMR can be problematic. Normal CRP and ESR results are observed in 14 and $27 \%$ of relapses respectively.(21) There is consequently an unmet need for a novel biomarker that accurately reflects the underlying inflammatory state in PMR. In research settings, the PMR-AS is considered a valid and reliable measure of disease activity; a value $\geq 9.35$ is associated with a sensitivity of $96.6 \%$ and specificity of $90.7 \%$ for flare diagnosis.(22) The composite nature of this scoring system is however less conducive to use in everyday clinical practice. In this study, a statistically significant relationship existed between PMR-AS and both NLR and PLR at baseline, with PLR also being found to correlate with disease activity during follow-up. We therefore propose that these indices, which can be simply calculated from an FBE, represent readily accessible and inexpensive biomarkers with the potential to measure PMR disease activity more reliably than conventional inflammatory markers like CRP and ESR. 
Several other studies have recently investigated trends in haematologic parameters in PMR. In their retrospective analysis of 94 PMR patients contrasted with 242 RA patients, Jung et al. found NLR and PLR to be significantly higher among PMR cases.(8) These levels subsequently diminished with treatment and were found to correlate with other measures of disease activity including CRP, however an association between NLR or PLR and a relapsing disease course was not identified in this instance. A Japanese study has however reported thrombocytosis as an adverse prognostic factor in PMR.(23) Using hierarchical cluster analysis, participants with a platelet count $>450 \times 10^{9 / L}$ were found to be less likely to exhibit a response to glucocorticoid therapy at one month. Finally, a change in leukocyte dynamics among patients with PMR and GCA compared with healthy and infection controls has been newly documented by van Sleen et al., with a shift towards the production of myeloid-lineage leukocytes noted prior to the commencement of prednisolone.(24) This bias ultimately persisted during treatment with a weaning schedule of glucocorticoid therapy and was even maintained in patients achieving drug-free remission.

Taken together, these results suggest that the inflammatory milieu which characterises PMR directly impacts neutrophil and platelet production within the bone marrow. As other authors have previously hypothesised, this likely occurs secondary to high levels of the key cytokine interleukin-6, given this proinflammatory mediator is additionally an independent regulator of granulopoiesis and stimulates thrombopoiesis through thrombopoietin. $(25,26)$ It is therefore entirely conceivable that haematologic parameters reported on an FBE may provide an accurate representation of underlying disease activity in PMR.

Whilst the precise mechanism behind the association of high NLR with adverse survival in cancer patients is not known, neutrophilia as an inflammatory response is understood to suppress other immune cells including lymphocytes, activated T cells and natural killer cells, whose infiltration of tumors is otherwise associated with positive cytotoxic treatment outcomes.(4) In RA, where a recent meta-analysis has demonstrated a consistent relationship between NLR and PLR, and the presence of active disease, these indices are similarly thought to reflect the pathogenic mechanisms at play rather than being surrogate measures of the body's overall inflammatory state.(27)

The pathogenesis of PMR remains comparatively unclear though - it is hypothesized to arise from an aberrant immune response that follows an interaction between environmental factors, possibly viral, and the innate immune system in genetically predisposed individuals.(28) Activated macrophages predominate in both synovial and arterial samples taken from PMR patients. In the related condition GCA however, neutrophils have been implicated as the promotors of an escaped proinflammatory disease phenotype.(29) More specifically, a subset of neutrophil granulocytes capable of suppressing T cell activity following the institution of high-dose prednisolone therapy have been demonstrated to lose this ability upon glucocorticoid weaning in the context of rising IL-17 and IL-6 levels. Loss of this neutrophil suppressor function is thought to result in unchecked $\mathrm{T}$ cell proliferation within the vessel wall and eventual disease relapse. 
Whilst this mechanism provides a biologically plausible explanation for a predominance of neutrophils enabling an adverse prognostic outcome in PMR, baseline neutrophil counts for glucocorticoid-resistant patients in our study were not substantially different from their glucocorticoid-responsive counterparts. Rather, lymphocyte counts were lower in this subset thereby producing higher NLR values. In general, no difference in total lymphocyte numbers is observed when PMR cases are compared with healthy controls. (30) Circulating B-cell lymphopaenia secondary to redistribution or intravascular marginalization of predominantly pro-inflammatory B-effector cells has however been established to exist in newly diagnosed, steroid-naïve PMR.(31) This disturbance in B cell homeostasis corrects with glucocorticoid therapy and is sustained in clinical remission but reoccurs upon disease relapse. Unfortunately, we did not undertake lymphocyte subset testing as part of this study, hence it is unclear whether lower lymphocyte counts at baseline in glucocorticoid-resistant patients represented an exaggeration of this previously described B cell phenomenon. Further investigation of lymphocyte subsets and their association with glucocorticoid resistance in PMR is now planned.

There are several other limitations to this study. It involves a relatively small number of participants and consequently the results may not be generalizable to a larger, more heterogenous PMR cohort. As glucocorticoids have the potential to impact upon measured neutrophil and platelet counts, it is similarly not known if NLR can predict glucocorticoid-resistant disease in PMR if calculated from an FBE obtained once the patient has commenced prednisolone. Finally, this study provides proof-of-concept for the utility of NLR and PLR in measuring PMR disease activity and predicting treatment response. For use in everyday practice however, cut-off values to aid clinical decision-making would be ideal. Unfortunately, this was beyond the scope of the current pilot and hence further validation of these biomarkers in a large, multi-center trial is necessary.

\section{Conclusion}

Baseline NLR represents a readily accessible and inexpensive biomarker that can predict glucocorticoid resistance in newly diagnosed PMR. This finding further characterizes a distinct subgroup of patients with relapsing disease in whom early initiation of DMARD therapy may be appropriate. In addition, both NLR and PLR appear to be reliable measures of PMR disease activity, outperforming conventional inflammatory markers like CRP and ESR. Further studies are now needed to validate these biomarkers for implementation in routine clinical practice.

\section{Abbreviations}


ACPA Anti-citrullinated peptide antibodies

\begin{tabular}{|c|c|}
\hline BMI & Body mass index \\
\hline BSR & British Society of Rheumatology \\
\hline CRP & C-reactive protein \\
\hline DMARD & Disease modifying anti-rheumatic drug \\
\hline EMS & Early morning stiffness \\
\hline ESR & Erythrocyte sedimentation rate \\
\hline FBE & Full blood examination \\
\hline${ }^{18}$ F-FDG & ${ }^{18}$ F-Fluorodeoxyglucose \\
\hline GCA & Giant cell arteritis \\
\hline HAQ-DI & Health assessment questionnaire-disability index \\
\hline IL & Interleukin \\
\hline MRI & Magnetic resonance imaging \\
\hline NLR & Neutrophil to lymphocyte ratio \\
\hline $\mathrm{PET} / \mathrm{CT}$ & Positron emission tomography/computed tomography \\
\hline PLR & Platelet to lymphocyte ratio \\
\hline PMR & Polymyalgia rheumatica \\
\hline PMR-AS & Polymyalgia rheumatica-activity score \\
\hline RA & Rheumatoid arthritis \\
\hline WCC & White cell count \\
\hline
\end{tabular}

\section{Declarations}

\section{Ethics Approval and Consent to Participate}

This study was approved by the Austin Health Human Research Ethics Committee (HREC/14/Austin/158) prior to commencement and all participants provided written consent prior to enrolment.

\section{Availability of Data and Materials}

The datasets used and analyzed during this study are available from the corresponding author on reasonable request. 
Author CO has received speaking honoraria from Roche, Janssen and Pfizer, and meeting sponsorship from Roche. Author CM declares no competing interests. Author DL declares no competing interests. Author $\mathrm{JL}$ has received a speaking honorarium from Abbvie and meeting sponsorship from Gilead. Author AS declares no competing interests. Author RB declares no competing interests.

\section{Funding}

Author $\mathrm{CO}$ has been supported by research grants received from Arthritis Australia and Austin Medical Research Foundation. Author $\mathrm{CO}$ has been supported by research grants received from Arthritis Australia and Austin Medical Research Foundation.

\section{Author Contributions}

Author CO designed the study, collected the patient data and was chiefly responsible for writing the manuscript. Author CM analyzed and interpreted all data presented. Author DL proposed further study of NLR and PLR in PMR and together with authors JL, AS and RB improved several iterations of the manuscript. All authors read and approved the final product.

\section{References}

1. Kremers HM, Reinalda MS, Crowson CS, Zinsmeister AR, Hunder GG, Gabriel SE. Relapse in a population based cohort of patients with polymyalgia rheumatica. J Rheumatol. 2005;32(1):65-73.

2. Dejaco C, Singh YP, Perel P, Hutchings A, Camellino D, Mackie S, et al. Current evidence for therapeutic interventions and prognostic factors in polymyalgia rheumatica: a systematic literature review informing the 2015 European League Against Rheumatism/American College of Rheumatology recommendations for the management of polymyalgia rheumatica. Ann Rheum Dis. 2015;74(10):1808-17.

3. Li B, Zhou P, Liu Y, Wei H, Yang X, Chen T, et al. Platelet-to-lymphocyte ratio in advanced Cancer: Review and meta-analysis. Clin Chim Acta. 2018;483:48-56.

4. Templeton AJ, McNamara MG, Seruga B, Vera-Badillo FE, Aneja P, Ocana A, et al. Prognostic role of neutrophil-to-lymphocyte ratio in solid tumors: a systematic review and meta-analysis. J Natl Cancer Inst.106(6):dju124.

5. Tekeoglu I, Gurol G, Harman H, Karakece E, Ciftci IH. Overlooked hematological markers of disease activity in rheumatoid arthritis. Int J Rheum Dis. 2016;19(11):1078-82.

6. Qin B, Ma N, Tang Q, Wei T, Yang M, Fu H, et al. Neutrophil to lymphocyte ratio (NLR) and platelet to lymphocyte ratio (PLR) were useful markers in assessment of inflammatory response and disease activity in SLE patients. Mod Rheumatol. 2016;26(3):372-6.

7. Hu ZD, Sun Y, Guo J, Huang YL, Qin BD, Gao Q, et al. Red blood cell distribution width and neutrophil/lymphocyte ratio are positively correlated with disease activity in primary Sjogren's syndrome. Clin Biochem. 2014;47(18):287-90. 
8. Jung JY, Lee E, Suh $\mathrm{CH}$, Kim HA. Neutrophil-to-lymphocyte ratio and platelet-to-lymphocyte ratio are associated with disease activity in polymyalgia rheumatica. J Clin Lab Anal. 2019:e23000.

9. Dasgupta B, Cimmino MA, Kremers HM, Schmidt WA, Schirmer M, Salvarani C, et al. 2012 Provisional classification criteria for polymyalgia rheumatica: a European League Against Rheumatism/American College of Rheumatology collaborative initiative. Arthritis Rheumatol. 2012;64(4):943-54.

10. Leeb BF, Bird HA. A disease activity score for polymyalgia rheumatica. Ann Rheum Dis. 2004;63(10):1279-83.

11. Dasgupta B, Borg FA, Hassan N, Barraclough K, Bourke B, Fulcher J, et al. BSR and BHPR guidelines for the management of polymyalgia rheumatica. Rheumatology. 2010;49(1):186-90.

12. Mori S, Koga Y, Ito K. Clinical characteristics of polymyalgia rheumatica in Japanese patients: evidence of synovitis and extracapsular inflammatory changes by fat suppression magnetic resonance imaging. Mod Rheumatol. 2007;17(5):369-75.

13. Team RC. R: A language and environment for statistical computing R Foundation for Statistical Computing, Vienna, Austria2013 [Available from: http://www.R-project.org/.

14. Goodrich B GJ, Ali I, Brilleman S rstanarm: Bayesian applied regression modeling via Stan 2018 [Available from: http://mc-stan.org/.

15. Vehtari A GJ, Yao Y, Gelman A. loo: Efficient leave-one-out cross-validation and WAIC for Bayesian models 2019 [Available from: http://CRAN.R-project.org/package=loo.

16. Morey RD HR, Rouder J Lee MD, Wagenmakers E. The fallacy of placing confidence in confidence intervals. . Psychon Bull Rev. 2016;23(1):103-23.

17. Ghosh J LY, Mitra R. On the use of cauchy prior distributions for Bayesian logistic regression Bayesian Anal. 2018;13(2):359-83.

18. Partington RJ, Muller S, Helliwell T, Mallen CD, Abdul Sultan A. Incidence, prevalence and treatment burden of polymyalgia rheumatica in the UK over two decades: a population-based study. Ann Rheum Dis. 2018;77(12):1750-6.

19. Hoes JN, Jacobs JW, Verstappen SM, Bijlsma JW, Van der Heijden GJ. Adverse events of low- to medium-dose oral glucocorticoids in inflammatory diseases: a meta-analysis. Ann Rheum Dis. 2009;68(12):1833-8.

20. Mackie SL, Pease CT, Fukuba E, Harris E, Emery P, Hodgson R, et al. Whole-body MRI of patients with polymyalgia rheumatica identifies a distinct subset with complete patient-reported response to glucocorticoids. Ann Rheum Dis. 2015;74(12):2188-92.

21. McCarthy EM, MacMullan PA, Al-Mudhaffer S, Madigan A, Donnelly S, McCarthy CJ, et al. Plasma fibrinogen is an accurate marker of disease activity in patients with polymyalgia rheumatica. Rheumatology. 2013;52(3):465-71.

22. Binard A, de Bandt M, Berthelot JM, Saraux A, Inflammatory Joint Disease Working Group of the French Society for R. Performance of the polymyalgia rheumatica activity score for diagnosing disease flares. Arthritis Rheumatol. 2008;59(2):263-9. 
23. Hayashi K, Ohashi K, Watanabe H, Sada KE, Shidahara K, Asano Y, et al. Thrombocytosis as a prognostic factor in polymyalgia rheumatica: characteristics determined from cluster analysis. Ther Adv Musculoskelet Dis. 2019;11:1759720X19864822.

24. van Sleen Y, Graver JC, Abdulahad WH, van der Geest KSM, Boots AMH, Sandovici M, et al. Leukocyte Dynamics Reveal a Persistent Myeloid Dominance in Giant Cell Arteritis and Polymyalgia Rheumatica. Front Immunol. 2019;10(1981).

25. Kaser A, Brandacher G, Steurer W, Kaser S, Offner FA, Zoller H, et al. Interleukin-6 stimulates thrombopoiesis through thrombopoietin: role in inflammatory thrombocytosis. Blood. 2001;98(9):2720-5.

26. Liu F, Poursine-Laurent J, Wu HY, Link DC. Interleukin-6 and the Granulocyte Colony-Stimulating Factor Receptor Are Major Independent Regulators of Granulopoiesis In Vivo But Are Not Required for Lineage Commitment or Terminal Differentiation. Blood. 1997;90(7):2583-90.

27. Erre GL, Paliogiannis P, Castagna F, Mangoni AA, Carru C, Passiu G, et al. Meta-analysis of neutrophilto-lymphocyte and platelet-to lymphocyte ratio in rheumatoid arthritis. Eur J Clin Invest.e13037.

28. Guggino G, Ferrante A, Macaluso F, Triolo G, Ciccia F. Pathogenesis of polymyalgia rheumatica. Reumatismo. 2018;70(1):10-7.

29. Nadkarni S, Dalli J, Hollywood J, Mason JC, Dasgupta B, Perretti M. Investigational analysis reveals a potential role for neutrophils in giant-cell arteritis disease progression. Circ Res. 2013;114(2):242-8.

30. Uddhammar A, Roos G, Nasman B, Dahlqvist SR. Peripheral blood lymphocyte subsets in polymyalgia rheumatica. Clin Rheumatol. 1995;14(1):62-7.

31. van der Geest KS, Abdulahad WH, Chalan P, Rutgers A, Horst G, Huitema MG, et al. Disturbed B cell homeostasis in newly diagnosed giant cell arteritis and polymyalgia rheumatica. Arthritis Rheumatol. 2014;66(7):1927-38.

\section{Figures}


Baseline Laboratory Values
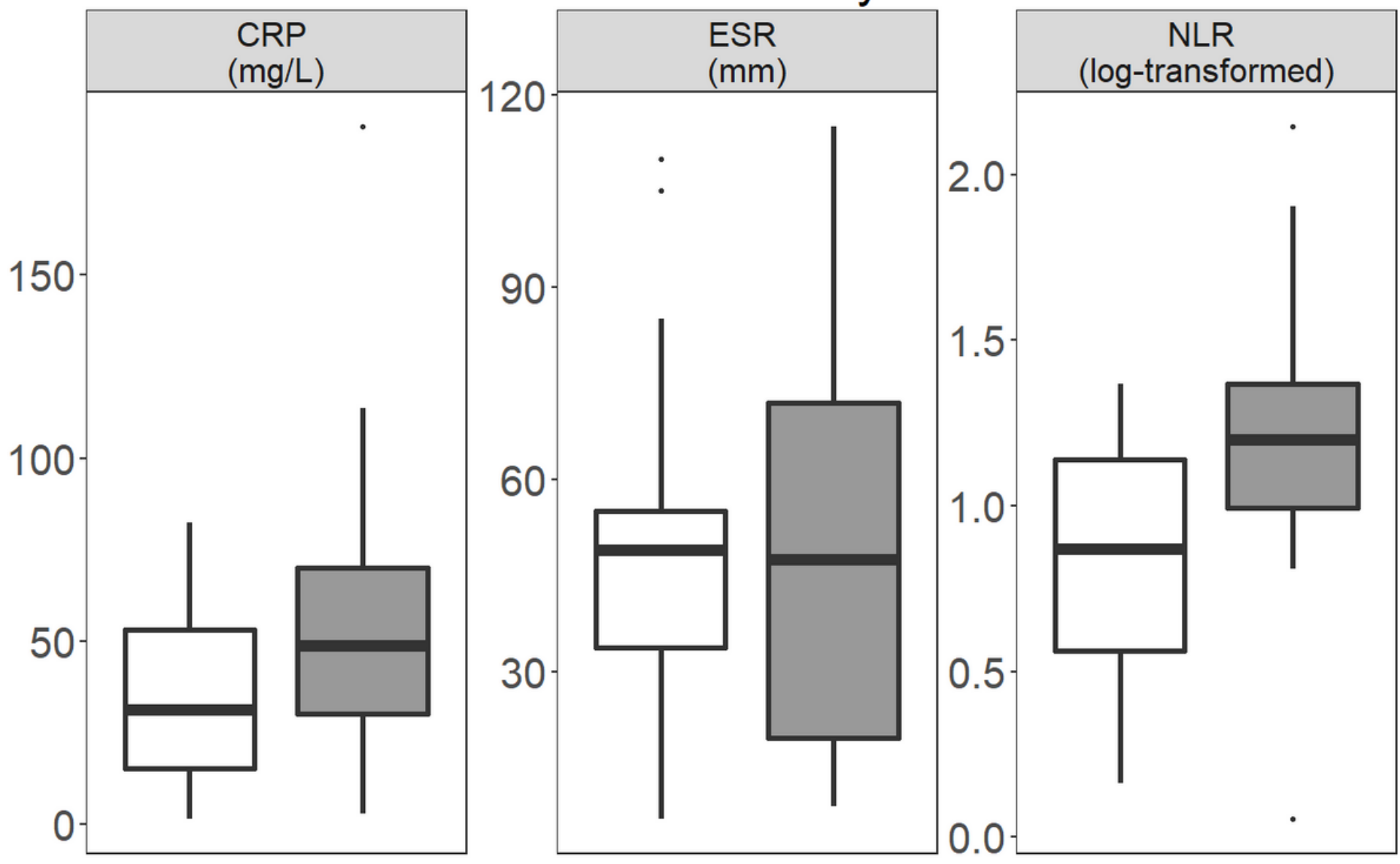

Glucocorticoid Resistant $\square$ No $\square$ Yes

Figure 1

Box plots: The relationship between baseline CRP, ESR and NLR values, and glucocorticoid-resistant disease. Abbreviations: CRP - C-reactive protein; ESR - erythrocyte sedimentation rate; NLR - neutrophil to lymphocyte ratio. 